\title{
Enriched homology and cohomology modules of simiplicial complexes
}

\author{
Gunnar Fløystad
}

Received: 9 August 2005 / Accepted: 5 September 2006 /

Published online: 5 October 2006

(C) Springer Science + Business Media, LLC 2006

\begin{abstract}
For a simplicial complex $\Delta$ on $\{1,2, \ldots, n\}$ we define enriched homology and cohomology modules. They are graded modules over $k\left[x_{1}, \ldots, x_{n}\right]$ whose ranks are equal to the dimensions of the reduced homology and cohomology groups.

We characterize Cohen-Macaulay, $l$-Cohen-Macaulay, Buchsbaum, and Gorenstein* complexes $\Delta$, and also orientable homology manifolds in terms of the enriched modules. We introduce the notion of girth for simplicial complexes and make a conjecture relating the girth to invariants of the simplicial complex.

We also put strong vanishing conditions on the enriched homology modules and describe the simplicial complexes we then get. They are block designs and include Steiner systems $S(c, d, n)$ and cyclic polytopes of even dimension.
\end{abstract}

Keywords Simplicial complex · Homology · Girth · Cohen-Macaulay · Simplicial complex · Block design · Homology manifold · Steiner system

\section{Introduction}

Given a simplicial complex $\Delta$ on the set $[n]=\{1,2, \ldots, n\}$ and a field $k$, one has its reduced homology groups $\tilde{H}_{i}(\Delta ; k)$ which depend only on the topological realization of $\Delta$. However the combinatorial structure makes $\Delta$ a richer object than its topological realization. In this paper we define enriched homology modules $\mathbf{H}_{i}(\Delta ; k)$ which are modules over the polynomial ring $k\left[x_{1}, \ldots, x_{n}\right]$, denoted by $S$. They have the property that the rank of $\mathbf{H}_{i}(\Delta ; k)$ as an $S$-module is equal to the dimension of $\tilde{H}_{i}(\Delta ; k)$ as a

This paper is to a large extent a complete rewriting of a previous preprint, "Hierarchies of simplicial complexes via the BGG-correspondence". Also Propositions 1.9 and 3.1 have been generalized to cell complexes in [11].

G. Fløystad $(\bowtie)$

Matematisk Institutt, Johs. Brunsgt. 12, 5008 Bergen, Norway

e-mail: gunnar@mi.uib.no 
vector space over $k$, and hence may be considered as an enrichment of the reduced homology groups.

The enriched homology modules are defined as the homology modules of the cellular complex, see [4], associated to $\Delta$ by attaching variable $x_{i}$ to the vertex $i$. Similarly we may define the enriched cohomology modules $\mathbf{H}^{i}(\Delta ; k)$ as the cohomology modules of the cocellular complex.

The classical criterion of Hochster for when a simplicial complex is CohenMacaulay, via the reduced homology of its links, translates in this setting to the criterion that the top enriched cohomology module $\mathbf{H}^{\operatorname{dim} \Delta}(\Delta ; k)$ is the only nonvanishing cohomology module. We also give a criterion for when $\Delta$ is Buchsbaum via its enriched cohomology modules.

The enriched cohomology modules turn out to be Alexander duals of the Matlis duals of the local cohomology modules of the Stanley-Reisner ring.

Hence they contain exactly the same information as these local cohomology modules. The enriched cohomology modules present another conceptual approach to this information, and the information appears in quite distinct algebraic forms. Therefore algebraic questions which are not interesting for local cohomology modules or their Matlis duals, turn out to be interesting for enriched cohomology modules.

For instance when $\Delta$ is Cohen-Macaulay we show in [11] that the top cohomology module can occur as an $l-1$ 'th syzygy module in an $S$-free resolution if and only if $\Delta$ is $l$-Cohen-Macaulay as defined by Baclawski [2]. (This notion means that $\Delta$ restricted to each subset $R$ of $[n]$ of cardinality $n+1-l$ is a Cohen-Macaulay complex of the same dimension as $\Delta$.)

For $l$-Cohen-Macaulay simplicial complexes, the dimension of $\Delta$ is less or equal to $n-1-l$ (unless $\Delta$ is the $n-l$-skeleton of the simplex), and the girth of $\Delta$, a suitable generalization of the well-known notion for graphs, is less or equal to $n+2-l$. We investigate $l$-Cohen-Macaulay simplicial complexes with one or more of these extremal values. In particular we make a conjecture concerning invariants of $l$-Cohen-Macaulay complexes of maximal girth, and prove the conjecture in the case where the dimension is submaximal equal to $n-1-l$.

Next we consider the case when the top cohomology module may be identified as an ideal in the polynomial ring $S$. When the top cohomology module is the only non-vanishing one, i.e. when $\Delta$ is $\mathrm{CM}$, we show that this happens exactly when $\Delta$ is a Gorenstein* simplicial complex. More generally for Buchsbaum complexes of positive dimension, we show that the top cohomology module is an ideal if and only if the complex is a connected orientable homology manifold.

In the end we put strong vanishing conditions on the enriched homology and cohomology modules and investigate what kind of simplicial complexes they corresponds to. For an $l$-Cohen-Macaulay $\Delta$ there is only one non-vanishing cohomology module. We require that there also be at most one non-vanishing homology module $\mathbf{H}_{i}(\Delta ; k)$ for $i<\operatorname{dim} \Delta$ (when $l \geq 2, \mathbf{H}_{\operatorname{dim} \Delta}(\Delta ; k)$ does not vanish), and that $\Delta$ has maximal girth, which is $n+2-l$. In [10] we introduced the notion of $\Delta$ being bi-Cohen-Macaulay, meaning that both $\Delta$ and its Alexander dual are Cohen-Macaulay. We show that the above vanishing condition is equivalent to the condition that the restriction of $\Delta$ to each subset $R$ of $[n]$ of cardinality $n+1-l$ is bi-Cohen-Macaulay of the same dimension and frame dimension as $\Delta$. (The frame dimension is by definition the dimension of the maximal complete skeleton of the simplex on $[n]$, which is contained in $\Delta$.) In 
this case the $f$-vector of $\Delta$ is completely determined by $l, n$, the dimension, and the frame dimension of $\Delta$, and we call $\Delta$ an l-Cohen-Macaulay design.

As examples, 1-Cohen-Macaulay designs are exactly the bi-Cohen-Macaulay simplicial complexes. $l$-Cohen-Macaulay designs of submaximal dimension $n-l-1$ are exactly the Alexander duals of Steiner systems $S(l-1, m, n)$ (where $m$ is related to the frame dimension of $\Delta$ ). 2-Cohen-Macaulay designs of dimension twice the frame dimension plus one, are examplified by the boundaries of cyclic polytopes of even dimension.

We also introduce the more general class of $(a, b)$-designs which specialize to $a+1-\mathrm{CM}$ designs when $b=0$, and show that they are block designs. As examples 2-neighbourly triangulations of surfaces are (1,1)-designs, and more generally homology manifolds with certain extremal behaviour of the Euler characteristic are $(1,1)$-designs.

The organization of the paper is as follows. In Section 1 we define the enriched homology and cohomology modules, and recall some basic facts on cellular complexes, Koszul duality and square free modules (as defined by Yanagawa [27]). We translate the link criterion of Hochster for $\Delta$ being Cohen-Macaulay, and the criterion of Schenzel for $\Delta$ being Buchbaum, to the setting of cohomology modules. We also translate the restriction criterion of Hochster for $\Delta$ being Cohen-Macaulay to the setting of homology modules.

In Section 2 we first recall a result from [11] providing motivation for the usefulness of enriched cohomology. Namely that the top cohomology module of CM complexes can occur as an $l-1$ 'th syzygy module in an $S$-free resolution iff $\Delta$ is $l$-CohenMacaulay. Then we go on to investigate $l$-CM simplicial complexes of maximal girth. In particular we make a conjecture concerning the invariants of such and prove this when the complex has submaximal dimension.

In Section 3 we consider when the top cohomology module is an ideal in the polynomial ring. For Cohen-Macaulay complexes this happens when it is Gorenstein*. More generally, for Buchsbaum complexes this happens when it is an orientable homology manifold.

In Section 4 we put strong vanishing conditions on the homology and cohomology modules and investigate the notion of $l$-Cohen-Macaulay designs. We also introduce the more general notion of $(a, b)$-designs. This section also contains many examples of such designs. Finally in Section 5 we give some problems and conjectures.

\section{Enriched homology and cohomology modules}

\subsection{Notation}

Denote by $[n]$ the set $\{1,2, \ldots, n\}$. A simplicial complex $\Delta$ on $[n]$ is a family of subsets of $[n]$ such that if $F$ is in $\Delta$ and $G$ is a subset of $F$, then $G$ is in $\Delta$.

An element $F$ in $\Delta$ is called a face of $\Delta$. If $F$ has cardinality $f$, its dimension is $f-1$. If $d$ is the maximal cardinality of a face, the dimension of $\Delta$ is $d-1$. A face of maximal cardinality is called a facet. The maximum $i$ such that all $i$-sets are in $\Delta$ is denoted by $c$ and we call $c-1$ the frame dimension of $\Delta$. 
If $R$ is a subset of $[n]$ denote by $\Delta_{R}$ the restricted simplicial complex on $R$, i.e. the complex consisting of the faces $F$ contained in $R$. It will also be convenient to have the notation $\Delta_{-R}$ for the restriction to the complement $[n] \backslash R$.

For $Q$ a subset of $[n]$ the link of $Q, \mathrm{k}_{\Delta} Q$, is the simplicial complex on $[n] \backslash Q$ consisting of the subsets $F$ of $[n] \backslash Q$ such that $Q \cup F$ is a face of $\Delta$.

A convention we will often use is that if a set is denoted by an upper case letter, say $R$, then the lower case letter $r$ will denote the cardinality of the set $R$.

\subsection{Enriched homology modules}

Given a field $k$, one has the augmented oriented chain complex $\tilde{\mathcal{C}}(\Delta ; k)$. The group $\tilde{\mathcal{C}_{i}}(\Delta ; k)$ is the vector space $\oplus k F$ with basis consisting of the faces of dimension $i$, and the differential defined by

$$
F \mapsto \sum_{\operatorname{dim} F^{\prime}=i-1} \epsilon\left(F, F^{\prime}\right) F^{\prime}
$$

where $\Delta \times \Delta \stackrel{\epsilon}{\longrightarrow}\{-1,0,1\}$ is a suitable incidence function (see [5]). The homology groups $\tilde{H}_{i}(\Delta ; k)$ of this complex are the reduced homology groups of $\Delta$ and depend only on the topological realization of $\Delta$.

However the combinatorial structure makes the simplicial complex a richer structure than its topological realization. We define enriched homology modules of the simplicial complex as follows. Let $S$ be the polynomial ring $k\left[x_{1}, \ldots, x_{n}\right]$. We get a complex $\mathcal{L}(\Delta$; $k$ ) of free $S$-modules by letting $\mathcal{L}_{i}(\Delta ; k)$ be the free $S$-module $\oplus S F$ with generators the faces of dimension $i$ and differential

$$
F \mapsto \sum_{F=F^{\prime} \cup\{l\}} \epsilon\left(F, F^{\prime}\right) x_{l} \cdot F^{\prime}
$$

We define the enriched homology modules $\mathbf{H}_{i}(\Delta ; k)$ (or just $\mathbf{H}_{i}(\Delta)$ ) to be the homology modules of this complex.

There are two sources of inspiration for this definition. First there is the theory of cellular complexes developed by Bayer, Peeva, and Sturmfels [3, 4]. The complex $\mathcal{L}(\Delta ; k)$ is the cellular complex obtained by attaching the monomial consisting of the single variable $x_{i}$ to vertex $i$. Another approach comes from the Koszul duality between the symmetric algebra $S$ and the exterior algebra $E$ in $n$ variables, and how this relates the module categories of these algebras, (see [8] and [10] for some recent articles). We explain this in some detail. This will also make the incidence function completely explicit.

Let $V$ be the vector space on generators $e_{1}, \ldots, e_{n}$ and

$$
E=E(V)=\oplus_{i=0}^{n} \wedge^{i} V
$$

be the exterior algebra. We let $W=V^{*}$ be the dual vector space with dual basis $x_{1}, \ldots, x_{n}$ and identify the polynomial $\operatorname{ring} S$ as the symmetric algebra $\operatorname{Sym}(W)$. We consider $V$ to have degree -1 and $W$ to have degree 1 . For a graded module $M$ over $E$ or $S$ we denote by $M(j)$ the module shifted $j$ steps to the left i.e. $M(j)_{d}=M_{j+d}$. 
Any graded (left) $E$-module $M$ gives rise to a complex of $S$-modules

$$
L(M): \cdots \rightarrow S \otimes_{k} M_{i} \stackrel{d_{i}}{\longrightarrow} S \otimes_{k} M_{i-1} \rightarrow \cdots
$$

where $d_{i}$ sends $s \otimes m$ to $\sum_{j} s x_{j} \otimes e_{j} m$. Note that the degree of $s \otimes m$ is the sum of the degrees of $s$ and $m$.

Given a simplicial complex $\Delta$, we can form the exterior face ring $k\{\Delta\}$, which is the quotient of $E$ by the monomial ideal $J_{\Delta}$ consisting of monomials $e_{i_{1}} \ldots e_{i_{r}}$ such that $\left\{i_{1}, \ldots, i_{r}\right\}$ is not a face of $\Delta$. Let $C_{\Delta}$ be the graded dual vector space of the exterior face ring $k\{\Delta\}$. It is a module over the exterior face ring. As a vector space it has a basis consisting of monomials $x_{i_{1}} \ldots x_{i_{r}}$ where $\left\{i_{1}, \ldots, i_{r}\right\}$ range over the faces of $\Delta$. Left multiplication by $e_{1}+e_{2}+\cdots+e_{n}$ gives a differential $d$ on $C_{\Delta}$ and the reduced homology of $\Delta$ is given by

$$
\tilde{H}_{p}(\Delta, k)=H_{p+1}\left(C_{\Delta}, d\right)
$$

The enriched homology of $\Delta$ is given by

$$
\mathbf{H}_{p}(\Delta ; k)=H_{p+1}\left(L\left(C_{\Delta}\right)\right) .
$$

We denote $L\left(C_{\Delta}\right)$ by $L(\Delta ; k)$ (or just $L(\Delta)$ ). Note that compared to $\mathcal{L}(\Delta ; k)$ it is shifted one step to the left.

If $\mathbf{b}$ is a multidegree in $\mathrm{N}^{n}$, the support of $\mathbf{b}$ is the set of non-vanishing coordinates. The following explicitly describes the multigraded parts of the homology module.

Lemma 1.1. For a multidegree $\mathbf{b}$ in $\mathbf{N}^{n}$, let $R$ be its support. Then

$$
\mathbf{H}_{p}(\Delta)_{\mathbf{b}} \cong \tilde{H}_{p}\left(\Delta_{R}\right) \text {. }
$$

Proof: This follows from the above description of $L(\Delta)$ as a cellular complex and the ideas in the proof of Proposition 1.1 in [4]. See also Corollary 1.6 below.

In particular we shall consider $\tilde{H}_{p}\left(\Delta_{R}\right)$ to have multidegree $R$.

Remark 1.2. From the above lemma and Hochster's description of the resolution of the Stanley-Reisner ring $k[\Delta]$, see [24] or originally [13], we see that the homology module $\mathbf{H}_{p}(\Delta)$ corresponds to the $p+1$ 'th linear strand in the resolution. Thus the collection of homology modules is equivalent to the linear strands of the resolution of the Stanley-Reisner ring. Our approach gives another point of view on this and new questions are natural to ask.

\subsection{Square free modules}

The notion of square free $S$-module was introduced by Yanagawa [27]. An $\mathbf{N}^{n}$-graded $S$-module $M$ is square free if $M_{\mathbf{b}} \stackrel{x_{i}}{\longrightarrow} M_{\mathbf{b}+u_{i}}$ is an isomorphism for every $\mathbf{b}$ in $\mathbf{N}^{n}$ with $i$ in the support of $\mathbf{b}$, and where $u_{i}$ is the $i$ 'th coordinate vector. 
It follows from the description of $L(\Delta)$ as a cellular complex, [4], that the enriched homology modules are square free $S$-modules. Part a. and b. in the following proposition are quotes and consequences of [27], Lemma 2.2, Corollary 2.4, and Proposition 2.5 .

Proposition 1.3. Let $M$ be a square free $S$-module.

a. The minimal prime ideals are the $\left(x_{i}\right)_{i \notin R}$ where $R$ are the maximal subsets of $[n]$ with nonzero $M_{R}$.

b. For $R$ maximal as above let $P$ be the corresponding prime ideal. There is a natural map $\left(M_{R}\right.$ has multidegree $\left.R\right)$

$$
S / P \otimes_{k} M_{R} \rightarrow M
$$

which becomes an isomorphism when localized at $P$.

The following is a justification for the term "enriched homology modules" enriched homology modules.

Corollary 1.4. The S-module rank of $\mathbf{H}_{p}(\Delta)$ is the dimension of $\tilde{H}_{p}(\Delta)$ as a vector space over $k$.

Proof: Let $M$ be $\mathbf{H}_{p}(\Delta)$ and $R=[n]$. Proposition 1.3 b. gives a natural map $S \otimes_{k}$ $\tilde{H}_{p}(\Delta) \rightarrow \mathbf{H}_{p}(\Delta)$ which becomes an isomorphism when localized at $(0)$.

There is also a notion of square free modules over the exterior algebra $E=$ $E\left(e_{1}, \ldots, e_{n}\right)$, namely a multigraded module $M$ is called square free if $M_{\mathbf{b}}$ is nonzero only if $\mathbf{b}$ is a characteristic vector of some $R \subseteq[n]$, i.e. a vector such that $b_{i}$ is 1 for $i$ in $R$ and $b_{i}$ is zero otherwise. (This notion is a variation of the one defined by Römer [22]. This is because we consider the $e_{i}$ to have negative degrees. According to our convention $E$ is not square free, but its dual $E(W)$ is.) We note that there is an equivalence of categories between the square free modules over $S$ and $E$ [22].

For a square free module $M$ over $E$, denote by $M_{\mid R}$ the restriction to $E_{R}=E\left(e_{i}, i \in\right.$ $R)$, i.e. $\left(M_{\mid R}\right)_{\mathbf{b}}$ is $M_{\mathbf{b}}$ when the support of $\mathbf{b}$ is in $R$, and zero otherwise. For a square free module over $S$ we may restrict it to $S_{R}=k\left[x_{i}, i \in R\right]$, the definition is by the same formulation as above. Now as in (1), define the functor $L_{R}$ on a module $M^{\prime}$ over $E_{R}$ to be

$$
L_{R}\left(M^{\prime}\right): \cdots \rightarrow S_{R} \otimes_{k} M_{i}^{\prime} \stackrel{d_{i}}{\longrightarrow} S_{R} \otimes_{k} M_{i-1}^{\prime} \rightarrow \cdots
$$

Lemma 1.5. Let $M$ be a square free module over $E$.

a. $L(M)_{\mid R}=L_{R}\left(M_{\mid R}\right)$.

b. $H^{p}(L(M))_{\mid R}=H^{p}\left(L_{R}\left(M_{\mid R}\right)\right)$.

\section{Proof:}

a. This is straightforward.

b. Follows since restriction is an exact functor. 
Corollary 1.6. $\mathbf{H}_{p}(\Delta)_{\mid R}=\mathbf{H}_{p}\left(\Delta_{R}\right)$.

Proof: This is because $\left(C_{\Delta}\right)_{\mid R}=C_{\Delta_{R}}$.

\subsection{Alexander duals}

The Alexander dual $\Delta^{*}$ of a simplicial complex $\Delta$, is the simplicial complex on $[n]$ consisting of the subsets $F$ such that $F^{c}$ is not in $\Delta$.

The Alexander dual of a square free module $M$ over $E\left(e_{1}, \ldots, e_{n}\right)$ is the square free module $M^{\vee}=\operatorname{Hom}_{k}(M, k(-[n]))$. Note that these notions are related by an exact sequence

$$
0 \rightarrow C_{\Delta^{*}} \rightarrow E(W) \rightarrow\left(C_{\Delta}\right)^{\vee} \rightarrow 0
$$

Let $M$ be a square free module over $S$. Via the equivalence of categories this corresponds to a square free module over $E$. We may take the Alexander dual of this module, and via the equivalence we again get a square free module $M^{*}$ over $S$, called the Alexander dual of $M$ [19] and [22]. Explicitly, for a subset $F$ of [n], $\left(M^{*}\right)_{F}$ is $\operatorname{Hom}_{k}\left(M_{F^{c}}, k\right)$ where $F^{c}$ is the complement of $F$ in [n]. If $i$ is not in $F$, the multiplication map

$$
\left(M^{*}\right)_{F} \stackrel{x_{i}}{\longrightarrow}\left(M^{*}\right)_{F \cup\{i\}}
$$

is the dual of the multiplication map

$$
M_{F^{c} \backslash\{i\}} \stackrel{x_{i}}{\longrightarrow} M_{F^{c}}
$$

By obvious extension this defines $\left(M^{*}\right)_{\mathbf{b}}$ for all $\mathbf{b}$ in $\mathbf{N}^{n}$ and all multiplications by variables.

\subsection{Enriched cohomology modules}

The reduced cohomology groups $\tilde{H}^{p}(\Delta ; k)$ of the simplicial complex $\Delta$ are the cohomology groups of the dualized complex

$$
\operatorname{Hom}_{k}(\tilde{\mathcal{C}}(\Delta ; k), k)
$$

We define the enriched cohomology modules $\mathbf{H}^{p}(\Delta ; k)$ (or just $\mathbf{H}^{p}(\Delta)$ ) as the cohomology modules of the dualized complex

$$
\mathcal{L}(\Delta ; k)^{\vee}=\operatorname{Hom}_{S}\left(\mathcal{L}(\Delta ; k), \omega_{S}\right)
$$

where $\omega_{S}$ is the canonical module of $S$, isomorphic to $S(-\mathbf{1})$ where $\mathbf{1}$ is the multidegree $(1,1, \ldots, 1)$. 
In terms of the Koszul duality correspondence this is given as follows. The reduced simplicial cohomology group is

$$
\tilde{H}^{p}(\Delta ; k)=H^{p+1}\left(\left(C_{\Delta}\right)^{*}\right) .
$$

The complex $\mathcal{L}(\Delta ; k)^{\vee}$ identifies as the complex $L\left(\left(\mathrm{C}_{\Delta}\right)^{\vee}\right)[-n]$ (here $[-n]$ denotes the complex shifted $n$ steps to the right) except that the former is shifted one step to the left. The enriched cohomology modules are

$$
\mathbf{H}^{p}(\Delta ; k)=H^{p+1}\left(L\left(\left(C_{\Delta}\right)^{\vee}\right)[-n]\right) .
$$

The following describes these cohomology modules in greater detail.

Proposition 1.7. $\mathbf{H}^{p}(\Delta)_{\mid R}=\mathbf{H}^{p-r^{c}}\left(l k_{\Delta} R^{c}\right)$. In particular $\mathbf{H}^{p}(\Delta)_{\mathbf{b}}$ is isomorphic to $\tilde{H}^{p-q}\left(l k_{\Delta} Q\right)$ where $Q$ is the complement in [n] of the support of $\mathbf{b}$.

Proof: This follows by Lemma 1.5 since $\left(C_{\Delta}\right)^{\vee}{ }_{\mid R}$ equals $\left(C_{\mathrm{lk}_{\Delta}} R^{c}\right)^{\vee}\left(-R^{c}\right)$.

The above corollary suggests that enriched cohomology modules are related to the local cohomology modules of the Stanley-Reisner ring. In fact, they contain exactly the same information as the following shows.

Theorem 1.8. $\mathbf{H}^{p}(\Delta)$ is the Alexander dual of $\operatorname{Ext}_{S}^{n-p-1}\left(k[\Delta], \omega_{s}\right)$, which again is Matlis dual to the local cohomology module $H_{m}^{p+1}(k[\Delta])$.

Proof: The last statement is a local duality. The former follows by [5, Theorem 5.6.3] or alternatively from [27, Proposition 3.1].

\subsection{Cohen-Macaulay and Buchsbaum simplicial complexes}

A simplicial complex $\Delta$ is called Cohen-Macaulay (CM) if the Stanley-Reisner ring $k[\Delta]$ is a Cohen-Macaulay ring. By a criterion of Hochster [13] or [24, II. 4], this is equivalent to

$$
\tilde{H}_{p}\left(\mathrm{lk}_{\Delta} R\right)=0 \text { for } p+r<\operatorname{dim} \Delta
$$

This gives the following criterion.

Proposition 1.9. $\Delta$ is Cohen-Macaulay iff the cohomology modules $\mathbf{H}^{p}(\Delta)$ vanish for $p<\operatorname{dim} \Delta$, or alternatively $L(\Delta)^{\vee}$ is a resolution of $\mathrm{H}^{\operatorname{dim} \Delta}(\Delta)$.

Proof: Since $k$ is a field, $\tilde{H}^{p}\left(\mathrm{lk}_{\Delta} R\right)$ is isomorphic to $\tilde{H}_{p}\left(\mathrm{lk}_{\Delta} R\right)^{*}$. By Proposition 1.7 and Hochster's criterion (3) this translates exactly to the above statement.

This shows that the well-known link criterion for $\Delta$ being Cohen-Macaulay is encoded quite compactly in the cohomology modules of $\Delta$. 
Remark 1.10. In general, when $k$ is not a field, the condition of only one non-vanishing cohomology module is weaker then being Cohen-Macaulay, only implication to the right holds in Proposition 1.9.

Proposition 1.11. When $\triangle$ is $C M$, the top cohomology module is the Alexander dual of the canonical module $\omega_{k[\Delta]}$ of the Stanley-Reisner ring.

Proof: By Theorem $1.8, \mathbf{H}^{\mathrm{dim} \Delta}(\Delta)$ is the Alexander dual to

$$
\operatorname{Ext}_{S}^{n-\operatorname{dim} \Delta-1}\left(k[\Delta], \omega_{S}\right)
$$

which is the canonical module of the Stanley-Reisner ring.

A simplicial complex $\Delta$ is called Buchsbaum if $k[\Delta]$ is a Buchsbaum ring. By a criterion of Schenzel [23] or [24, II.8], this is equivalent to

$$
\tilde{H}_{i}\left(\mathrm{lk}_{\Delta} R\right)=0 \text { for } i+r<\operatorname{dim} \Delta, r \geq 1 .
$$

This gives the following criterion.

Proposition 1.12. $\Delta$ is Buchsbaum iff

$$
\mathbf{H}^{p}(\Delta) \cong S \otimes_{k} \tilde{H}^{p}(\Delta) \quad \text { for } p<\operatorname{dim} \Delta
$$

(Note that $\tilde{H}^{p}(\Delta)$ has multidegree $\mathbf{1}$, see convention after Lemma 1.1.)

Proof: When $p<\operatorname{dim} \Delta$ the criterion (4) says that $\mathbf{H}^{p}(\Delta)_{\mathbf{b}}$ vanishes unless the support of $\mathbf{b}$ is the whole of $[n]$. But any square free module $M$ with this property must be of the form $S \otimes_{k} M_{[n]}$.

An alternative criterion for the Cohen-Macaulayness of $\Delta$ is the following, [13, p. 197],

$$
\tilde{H}_{p}\left(\Delta_{-R}\right)=0 \text { for } p+r<\operatorname{dim} \Delta
$$

This follows directly from Hochster's formula for the multigraded Tor in the resolution of the Stanley-Reisner ring.

For a square free module $M$, the codimension of $M$ is $\geq c$ iff $M_{[n] \backslash F}$ is zero for all $F$ of cardinality less than $c$. Hochster's criterion (5) is then equivalent to the following.

Proposition 1.13. $\Delta$ is $C M$ iff each homology module $\mathbf{H}_{\operatorname{dim} \Delta-i}(\Delta)$ has codimension $\geq i$. 


\section{2 l-Cohen-Macaulay simplicial complexes}

For Cohen-Macaulay simplicial complexes there is only one nonvanishing cohomology module, $\mathbf{H}^{\operatorname{dim} \Delta}(\Delta)$. It is therefore natural to put various algebraic conditions on this module and investigate what properties they correspond to for a simplicial complex.

\section{1 -CM simplicial complexes}

In [2], Baclawski introduced the notion of l-Cohen-Macaulay simplicial complexes, which geometrically corresponds to higher connectivity. A simplicial complex $\Delta$ is said to be $l$-Cohen-Macaulay $(l-\mathrm{CM})$ if $\Delta_{-R}$ is Cohen-Macaulay of the same dimension as $\Delta$, for all $R$ of cardinality $\leq l-1$. For instance if $\Delta$ is a graph, then $\Delta$ is $l$-CM iff it is (vertex) $l$-connected.

In [11] we prove that this property has nice descriptions in terms of the top cohomology module and also in terms of the homology modules, generalizing Propositions 1.9 and 1.13 .

Theorem 2.1 ([11]). The following are equivalent for a simplicial complex $\Delta$.

1. $\Delta$ is $C M$ and $\mathbf{H}^{\operatorname{dim} \Delta}(\Delta)$ can occur as an $l-1$ 'th syzygy module in an $S$-free resolution.

2. The codimension of $\mathbf{H}_{\mathrm{dim} \Delta-i}(\Delta)$ is greater than or equal to $(l-1)+i$ for $i \geq 1$.

3. $\Delta$ is $l-C M$.

Remark 2.2. In the theory of polytopes, Balinski's theorem [28], says that the 1 -skeleton of a $d$-dimensional polytope is $d$-connected. In [11] we show a comprehensive generalization of Balinski's theorem, namely that the codimension $r$ skeleton of an $l$-CM simplicial complex is $l+r$-CM. This is a rather immediate consequence of the above theorem.

Remark 2.3. From [2] we mention the following two properties which produce new $l$-CM complexes. (i) If $\Delta$ is an $l$-CM simplicial complex, then any link $\mathrm{lk}_{\Delta} Q$ is $l$-CM. (ii) If $\Delta_{1}$ and $\Delta_{2}$ are $l$-CM simplicial complexes, their join $\Delta_{1} * \Delta_{2}$ is $l$-CM. In particular since $l$ vertices is $l$-CM, if $\Delta$ is $l$-CM, the $l$-point suspension of $\Delta$ is l-CM.

\subsection{Maximal girth and maximal dimension}

For a simplicial complex $\Delta$ we define its girth to be the smallest degree in which the top homology module $\mathbf{H}_{\operatorname{dim} \Delta}(\Delta)$ is nonzero. Since homology modules are square free the girth is $\leq n$ provided the top homology module does not vanish. If it vanishes we define the girth to be $n+1$.

If $\Delta$ is a graph, this specializes to a notion of a girth for graphs, the length of a cycle of minimal length. 


\section{Proposition 2.4. Let a non-empty $\Delta$ be l-CM}

a. Its girth $\leq n+2-l$.

b. The cardinality $d$ of a facet is $\leq n-l$, unless $\Delta$ is the $n-l$-skeleton of the $n-1$ simplex.

\section{Proof:}

a. We want to show that $H_{\operatorname{dim} \Delta}\left(\Delta_{-R}\right)$ is non-zero for some $R$ of cardinality $l-2$. By restricting to $\Delta^{\prime}=\Delta_{-R}$ for some $R$ of cardinality $l-2$, it will be sufficient to show this for 2-CM $\Delta^{\prime}$. Now it is easy to see that the top cohomology module of any simplicial complex is non-zero unless the simplicial complex is empty. For instance this may be seen from Proposition 1.7 by taking $Q$ to be a facet of a simplicial complex. But then applying Theorem 2.1 to the 2-CM $\Delta^{\prime}$, its non-zero enriched top cohomology module $\mathbf{H}^{\operatorname{dim} \Delta^{\prime}}\left(\Delta^{\prime}\right)$ must have rank $\geq 1$ as an $S$-module. Then $\tilde{H}^{\operatorname{dim} \Delta^{\prime}}\left(\Delta^{\prime}\right)$ is nonzero and so also is the reduced homology module $\tilde{H}_{\text {dim } \Delta^{\prime}}\left(\Delta^{\prime}\right)$.

b. The restriction $\Delta_{-R}$ has the same dimension as $\Delta$ for all $R$ of cardinality $l-1$. Hence $d \leq n+1-l$. If $d$ were $n+1-l$, each $\Delta_{-R}$ would be a simplex, since $[n] \backslash R$ has cardinality $n+1-l$. But then $\Delta$ would be the $n-l$-skeleton of the simplex on $[n]$.

Remark 2.5. If $\Delta$ is $l$-CM, its codimension one skeleton has girth $\leq d$, since we delete the interiors of the facets. Now if $\Delta$ is not the $n-l$-skeleton, we have $d \leq n-l$. Thus its codimension one skeleton, which is $l+1$-Cohen-Macaulay, will not have maximal girth.

The following characterizes those simplicial complexes attaining the submaximal $d$. Actually the characterization is more transparently given in terms of the Alexander dual $\Delta^{*}$. Recall that a missing face $F$ is a subset of $[n]$ not in $\Delta$. Also the frame dimension of $\Delta^{*}$ is denoted $c^{*}-1$.

Proposition 2.6. The following are equivalent for a simplicial complex $\Delta$.

a. $\Delta$ is $l-C M$ with d equal to $n-l$.

b. $d$ is $n-l$ and the cardinality of $F \cup G$ is $\geq n+2-l$ for any two distinct minimal missing faces.

c. $c^{*}$ is $l-1$ and any two distinct facets of $\Delta^{*}$ intersect in a subset of cardinality less than $l-1$.

The girth of $\Delta$ being maximal, i.e. $n+2-l$ corresponds to the cardinality of any minimal missing face being $\leq n-l$, respectively every facet of $\Delta^{*}$ having cardinality $\geq l$.

Proof: The equivalence of $b$. and $c$. is clear since $F$ is a minimal missing face of $\Delta$ iff the complement $F^{c}$ is a facet of $\Delta^{*}$, and $c^{*}+d+1$ is $n$.

$a$. $\Rightarrow b$. Assume $\Delta$ is $l$-CM with $d$ equal to $n-l$. Let $R$ be a subset of cardinality $l-1$. Then $\Delta^{\prime}=\Delta_{-R}$ is CM with $d$ equal to $n-l=: n^{\prime}-1$. We must show that $\Delta^{\prime}$ 
has only one minimal missing face. But this is true, as the only minimal missing face is the intersection of the missing faces of cardinality $n^{\prime}-1$.

Before proving the converse, note that if $\Delta$ is a simplicial complex containing only one minimal missing face $F$, then $F$ is non-empty. Letting $x$ be in $F$, the restriction $\Delta_{-\{x\}}$ contains no minimal missing face and so is a simplex and therefore $d$ is $n-1$.

$b$. $\Rightarrow a$. Let $d$ be $n-l$ with $l \geq 1$ and $R$ have cardinality $l-1$. Then $\Delta^{\prime}=\Delta_{-R}$ contains at most one minimal missing face. Since $d^{\prime} \leq n-l$, by the above it contains exactly one minimal missing face $F$ and the facets are exactly the $n^{\prime}-1$-sets of $[n] \backslash R$ not containing $F$. Therefore $\Delta^{\prime}$ is $\mathrm{CM}$ of the same dimension as $\Delta$, and so $\Delta$ is $l$-CM with $d$ equal to $n-l$.

To prove the last statement, note that any minimal missing face of $\Delta$ has cardinality $\leq d+1$ which is $n+1-l$. That the girth of $\Delta$ is maximal, i.e. $n+2-l$ means that $\tilde{\tilde{H}}_{\text {dim } \Delta}\left(\Delta_{-R}\right)$ which is $\tilde{H}_{n-l-1}\left(\Delta_{-R}\right)$ vanishes when $R$ is of cardinality $l-1$. But then $[n] \backslash R$ is not a minimal missing face and so all these have cardinality $\leq n-l$.

Remark 2.7. When $l$ is 2 we see that for the Alexander dual of a 2-CM simplicial complex $\Delta$ with $d$ submaximal equal to $n-2$, the facets partition $[n]$ into disjoint subsets. In general for an $l$-CM complex with $d$ equal to $n-l$, the facets of its Alexander dual form a collection of subsets $F_{1}, \ldots, F_{m}$ such that each $l-1$-subset is contained in exactly one subset $F_{i}$. The girth of $\Delta$ is $n+2-l$ if all the $F_{i}$ have cardinality $\geq l$.

If $\Delta$ is 3-CM and if $d$ is 2, i.e. $\Delta$ is a graph which is 3-connected, it is rather clear that if $\Delta$ has a reasonably large number of vertices, then $\Delta$ cannot have maximal girth $n-1$. This suggests that for $l$-CM $\Delta$ of maximal girth and $n$ reasonably large, the dimension will not be too small. This would be a consequence of the following.

Conjecture 2.8. Let $\Delta$ be an $l$-CM simplicial complex of maximal girth. Assume it is not the $r$-skeleton of the $l+r-1$-simplex for some $r$. Then $c \geq l-1$.

This of course implies that $d \geq l$ if $\Delta$ is not the skeleton of some simplex of dimension $\leq 2 l-3$.

We prove this conjecture in the case of $d$ submaximal equal to $n-l$. In fact we prove something stronger.

Proposition 2.9. Let $\Delta$ be an l-CM simplicial complex of maximal girth with d equal to $n-l$. Then $c \geq l-1$ and for $l \geq 3$ and $n \geq 3 l-4$ we have the stronger bound $c \geq(n+2-l) / 2$.

Proof: Clearly the statement holds if $l$ is 1 or 2 . So we assume $l \geq 3$ and consider the Alexander dual simplicial complex $\Delta^{*}$. Let $X$ be a facet of $\Delta^{*}$ of cardinality $d^{*}$, which is $\geq l$ by Proposition 2.6, and let $Y$ be the complement $[n] \backslash X$. We shall count the number of pairs $(A, B)$ where:

i. $A \subseteq X$ has cardinality $l-2$,

ii. $B \subseteq Y$ has cardinality 2 ,

iii. $A \cup B$ is a face of $\Delta^{*}$.

First assume we are given $A$ fulfilling i. Since $c^{*}=l-1$, we may take any $y$ in $Y$ and $A \cup\{y\}$ will be a face. By Proposition 2.6, it may be extended to a face $A \cup\{y, z\}$. 
Now $z$ cannot be in $X$ since any two facets of $\Delta^{*}$ intersect in cardinality $\leq l-2$. Hence $z$ is in $Y$. This gives that the number of pairs $(A, B)$ fulfilling i., ii., and iii. is greater than or equal to

$$
\frac{n-d^{*}}{2} \cdot\left(\begin{array}{c}
d^{*} \\
l-2
\end{array}\right)
$$

Now given $B$ fulfilling ii, consider the set of $A$ 's fulfilling i. and iii. If $A_{1}$ and $A_{2}$ are such two distinct sets, then the union of $A_{1}, A_{2}$ and $B$ is not a face, since it would intersect $X$ in a set of cardinality $\geq l-1$. Hence $A_{1} \cup B$ and $A_{2} \cup B$ are in distinct facets and so $A_{1}$ and $A_{2}$ intersect in a set of cardinality $\leq l-4$. Thus each $l-3$-set in $X$ is contained in at most one $A$. So for a given $B$ the number of possible $A$ 's is less than or equal to

$$
\frac{1}{l-2} \cdot\left(\begin{array}{c}
d^{*} \\
l-3
\end{array}\right)
$$

Summing over the $B$ 's, the number of pairs $(A, B)$ fulfilling i., ii., and iii. is less than or equal to

$$
\left(\begin{array}{c}
n-d^{*} \\
2
\end{array}\right) \cdot \frac{1}{l-2} \cdot\left(\begin{array}{c}
d^{*} \\
l-3
\end{array}\right)
$$

This implies that (6) is less than or equal to (8) which gives

$$
d^{*}-l+3 \leq n-d^{*}-1
$$

Putting $d^{*}=n-c-1$ this becomes

$$
c \geq \frac{n+2-l}{2}
$$

Now let $\bar{A}$ be the complement of $A$ in $X$, of cardinality $d^{*}-l+2$. For a given $B$ any two distinct $\bar{A}_{1}$ and $\bar{A}_{2}$ intersect in a set of cardinality $\leq d^{*}-l$. Thus the number of pairs $(\bar{A}, B)$ where $(A, B)$ fulfills i., ii., and iii. is less or equal to

$$
\left(\begin{array}{c}
n-d^{*} \\
2
\end{array}\right) \cdot \frac{1}{d^{*}-l+2} \cdot\left(\begin{array}{c}
d^{*} \\
d^{*}-l+1
\end{array}\right) .
$$

This implies that (6) is less than or equal to (9) which gives

$$
1 \leq \frac{1}{d^{*}-l+2} \cdot \frac{d^{*}-l+2}{l-1} \cdot\left(n-d^{*}-1\right) .
$$

Putting $d^{*}=n-c-1$ this becomes

$$
c \geq l-1 \text {. }
$$




\section{Gorenstein* complexes and orientable homology manifolds}

Another natural question concerning the top cohomology module of CM-complexes is when it can be identified as an ideal in $S$, or more intrinsically as a rank one torsion free module over $S$. The answer is that this happens exactly when $\Delta$ is Gorenstein*. More generally we show that for Buchsbaum complexes it happens when the complex is an orientable homology manifold.

\subsection{Gorenstein* complexes}

Recall that $\Delta$ is a Gorenstein simplicial complex iff the Stanley-Reisner ring $k[\Delta]$ is a Gorenstein ring. If $\Delta$ is also not a cone, it is called Gorenstein*. By a criterion of Hochster this latter is equivalent to $\Delta$ being $\mathrm{CM}$ and $\tilde{H}_{p}\left(\mathrm{lk}_{\Delta} R\right)=k$ whenever $p+r=\operatorname{dim} \Delta$ and $R$ is a face of $\Delta$.

Theorem 3.1. Let $\Delta$ be CM. The top cohomology module of $\Delta$ is a rank one torsion free $S$-module iff $\Delta$ is a Gorenstein* simplicial complex. The top cohomology module then identifies as the Stanley-Reisner ideal (in S) of the Alexander dual of $\Delta$.

Proof: Note first that if $M$ is square free then $M^{\vee}=\operatorname{Hom}_{S}\left(M, \omega_{S}\right)$ is also square free as can be seen by taking a free presentation of $M$.

Consider then a non-zero map $S(-\mathbf{a}) \rightarrow \mathbf{H}^{\operatorname{dim} \Delta}(\Delta)^{\vee}$ where $S(-\mathbf{a})$ is square free. Dualized this gives a nonzero composition

$$
\mathbf{H}^{\operatorname{dim} \Delta}(\Delta) \rightarrow \mathbf{H}^{\operatorname{dim} \Delta}(\Delta)^{\vee \vee} \rightarrow S\left(-\mathbf{a}^{c}\right) \rightarrow S
$$

If the top cohomology module is torsion free of rank one this map is an inclusion. If we take the Alexander dual we get a surjection

$$
S \rightarrow \operatorname{Ext}_{S}^{n-d}\left(k[\Delta], \omega_{S}\right)
$$

The module on the right-hand-side of $(11)$ is a $k[\Delta]$-module and must therefore be a quotient of $k[\Delta]$. Since $k[\Delta]$ is unmixed, the right side of (11) has the same asscoiated prime ideals, see [26] Proposition 3.6.b on p. 51. Therefore since $k[\Delta]$ is reduced they must be equal. Thus $k[\Delta]$ becomes Gorenstein and Gorenstein* since $\Delta$ is not acyclic.

Conversely if $\Delta$ is Gorenstein* the right-hand-side of (11) is $k[\Delta]$. The Alexander dual of (11) then identifies the top cohomology module as the ideal $I_{\Delta^{*}}$ in $S$.

Remark 3.2. When $\Delta$ is zero-dimensional, i.e. a set of vertices, $\Delta$ is $l$-CM if $\Delta$ consists of $l$ or more vertices. Thus if $\Delta$ is an $l$-CM simplicial complex any link $\operatorname{lk}_{\Delta} Q$ which is a non-empty point set will consist of $l$ or more points. When $l$ is equal to 2, Gorenstein* complexes are the 2-CM complexes where each such link consists of exactly two points. Hence the class of $l$-CM complexes in which the links that are 祭Springer 
non-empty point sets consist of exactly $l$ points might be a reasonable generalization of Gorenstein* simplicial complexes.

Theorem 3.3. Let $\Delta$ be a Gorenstein* simplicial complex. The homology modules $\mathbf{H}_{i}(\Delta)$ and $\mathbf{H}_{\mathrm{dim} \Delta-1-i}(\Delta)$ are Alexander dual square free modules.

Proof: In the resolution of $k[\Delta]$, in each linear strand the differential is given by maps [13],

$$
S \otimes_{k} \tilde{H}^{p}\left(\Delta_{R \cup\{i\}}\right) \rightarrow S \otimes_{k} \tilde{H}^{p}\left(\Delta_{R}\right)
$$

When $\Delta$ is Gorenstein*, the resolution of $k[\Delta]$ is self-dual and $\operatorname{Hom}_{S}\left(-, \omega_{S}\right)$ of $(12)$ identifies as

$$
S \otimes_{k} \tilde{H}^{\operatorname{dim} \Delta-1-p}\left(\Delta_{-R}\right) \rightarrow S \otimes_{k} \tilde{H}^{\operatorname{dim} \Delta-1-p}\left(\Delta_{-R-\{i\}}\right) .
$$

Hence the natural maps

$$
\tilde{H}_{p}\left(\Delta_{R}\right) \rightarrow \tilde{H}_{p}\left(\Delta_{R \cup\{i\}}\right)
$$

and

$$
\tilde{H}_{\operatorname{dim} \Delta-1-p}\left(\Delta_{-R-\{i\}}\right) \rightarrow \tilde{H}_{\operatorname{dim} \Delta-1-p}\left(\Delta_{-R}\right)
$$

are dual to each other. But this means that the square free modules $\mathbf{H}_{i}(\Delta)$ and $\mathbf{H}_{\mathrm{dim} \Delta-1-i}(\Delta)$ are Alexander duals.

\subsection{Orientable homology manifolds}

Recall that a connected $\Delta$ is an orientable homology manifold if all proper links of $\Delta$ are Gorenstein* and $\tilde{H}_{\operatorname{dim} \Delta}(\Delta)$ is $k$. Then in particular $\Delta$ is Buchsbaum.

Theorem 3.4. Let $\Delta$ be Buchsbaum of dimension $\geq 1$. Then $\Delta$ is a connected orientable homology manifold iff the top cohomology module is a rank one torsion free $S$-module. It may be identified as the Stanley-Reisner ideal (in S) of the Alexander dual of $\Delta$.

Proof: If the top cohomology module is torsion free of rank one we get as in Theorem 3.1 an inclusion

$$
\mathbf{H}^{\operatorname{dim} \Delta}(\Delta) \rightarrow S
$$

Taking the Alexander dual we get a surjection

$$
S \rightarrow \operatorname{Ext}_{S}^{n-d}\left(k[\Delta], \omega_{S}\right) .
$$


The module on the right-hand-side of (14) is a $k[\Delta]$-module and as such a quotient of $k[\Delta]$. Also it has the same associated prime ideals as $k[\Delta]$ since the latter is unmixed, see [26], Proposition 3.6.b on p. 51 and Corollary 2.4 on p. 75 . Since $k[\Delta]$ is reduced the right hand side of (14) must then be equal to $k[\Delta]$. Taking the Alexander dual of (14) we get that the left-hand-side of (13) identifies as $I_{\Delta^{*}}$.

Since $\mathbf{H}^{p}\left(\mathrm{k}_{\Delta}\{x\}\right)$ is $\mathbf{H}^{p}(\Delta)_{\mid[n] \backslash\{x\}}$ by Proposition 1.7 , it follows by Theorem 3.1 that $\operatorname{lk}_{\Delta}\{x\}$ is Gorenstein*. Therefore $\Delta$ is an orientable homology manifold.

Conversely suppose $\Delta$ is a connected orientable homology manifold. Then $\mathbf{H}^{\operatorname{dim} \Delta}(\Delta)$ has rank one. If it had torsion there would have to be a proper subset $R$ of $[n]$ such that $\mathbf{H}^{\operatorname{dim} \Delta}(\Delta)_{\mid R}$ had rank $\geq 2$. But since this module identifies as $\mathbf{H}^{\operatorname{dim} \Delta-r^{c}}\left(\operatorname{lk}_{\Delta} R^{c}\right)$ and this link is Gorenstein*, this is impossible. Thus the top cohomology module is torsion free of rank one.

\section{Designs and vanishing of homology modules}

Assume $\Delta$ is not $\emptyset$ or the simplex on $[n]$. Recall that $c$ is the maximum integer $i$ such that all $i$-sets are in $\Delta$. If $T$ is a $c+1$-set not in $\Delta$, then $\tilde{H}_{c-1}\left(\Delta_{T}\right)$ is non-zero. Hence the homology module $\mathbf{H}_{c-1}(\Delta)$ is non-zero. The following, from [10], describes when the other homology modules vanish.

\subsection{Bi-Cohen-Macaulay complexes}

Theorem 4.1. There is at most one non-vanishing homology module $\mathbf{H}_{i}(\Delta)$ (with the exception of $\mathbf{H}_{-1}(\Delta)$ if this is $k$ ) iff the Alexander dual of $\Delta$ is Cohen-Macaulay.

Remark 4.2. $\mathbf{H}_{-1}(\Delta)$ is $k$ iff all vertices of $[n]$ are in $\Delta$.

When both $\Delta$ and $\Delta^{*}$ are Cohen-Macaulay we call $\Delta$ bi-Cohen-Macaulay. This corresponds to $\Delta$ having one non-vanishing cohomology module and one non-vanishing homology module (save the exception).

Example 4.3. When $\Delta$ is a graph, $\Delta$ is Cohen-Macaulay iff it is connected. The Alexander dual of $\Delta$ is Cohen-Macaulay iff $\Delta$ is a forest. Hence $\Delta$ is bi-CohenMacaulay iff it is a tree.

In [10] it was shown that the $f$-vector of a bi-Cohen-Macaulay simplicial complex only depends on the number of vertices $n$, its dimension, and its frame dimension. If $f_{\Delta}(t)=\sum f_{i-1} t^{i}$ is the $f$-polynomial then

$$
f_{\Delta}(t)=(1+t)^{d-c}\left(1+(n-d+c) t+\cdots+\left(\begin{array}{c}
n-d+c \\
c
\end{array}\right) t^{c}\right) .
$$




\section{2 l-Cohen-Macaulay designs}

Our objective is now to put strong vanishing conditions on the homology modules of $l$-CM simplicial complexes and investigate what kind of simplicial complexes we obtain this way.

We define a simplicial complex to be an $l$-CM design iff (i) $\Delta_{-R}$ is bi-CM of the same dimension and frame dimension as $\Delta$ for all $R$ of cardinality $l-1$ and (ii) $\Delta$ is not the $(n-p)$-skeleton of a simplex for some $p>l$.

Theorem 4.4. $\Delta$ is an l-CM design iff $\Delta$ is $l$-CM, of maximal girth $n+2-l$, and has at most one non-vanishing homology module $\mathbf{H}_{i}(\Delta)$ for $i<\operatorname{dim} \Delta$ (with the exception of $\mathbf{H}_{-1}(\Delta)$ if this is $\left.k\right)$.

Proof: First assume $\Delta$ is an $l$-CM design. If $d>c$, then $\tilde{H}_{\operatorname{dim} \Delta}\left(\Delta_{-R}\right)$ vanishes for $R$ of cardinality $l-1$. So $\mathbf{H}_{\operatorname{dim} \Delta}(\Delta)$ is zero in degrees $\leq n+1-l$ and the girth of $\Delta$ is $n+2-l$. This is also true if $d$ (and hence $c$ ) is $n+1-l$.

Since $\Delta$ is $l$-CM, $\tilde{H}_{i}\left(\Delta_{-R}\right)$ vanishes when $i+r \leq d+l-3$ and $i \leq d-2$. In particular when $r \leq l-1$ and $i \leq d-2$, it vanishes. When $i$ is not equal to $c-1$ and $r \geq l$ it will also vanish as we now explain. Let $T$ be a subset of $R$ of cardinality $l-1$. $\Delta_{-T}$ is bi-CM of frame dimension $c-1$, and so $\mathbf{H}_{i}\left(\Delta_{-T}\right)$ vanishes for $i$ not equal to $c-1$ (except for $i=-1$ when this homology module is $k$ ). Hence $\mathbf{H}_{i}(\Delta)$ vanishes for $i<\operatorname{dim} \Delta$, except for $i$ equal to $c-1$ (and for $i=-1$ when this homology module is $k$ ).

Conversely, suppose $\Delta$ is $l$-CM, has maximal girth $n+2-l$ and at most one nonvanishing $\mathbf{H}_{i}(\Delta)$ for $i<\operatorname{dim} \Delta$ (save the exception). If $\Delta$ is a skeleton of the simplex, the condition of maximal girth clearly implies that $\Delta$ is the $n-l$-skeleton.

Let $R$ be a subset of $[n]$ of cardinality $l-1$. Then $\mathbf{H}_{i}(\Delta)_{\mathbf{b}}$ is equal to $\mathbf{H}_{i}\left(\Delta_{-R}\right)_{\mathbf{b}}$ when $\mathbf{b}$ has support in $[n] \backslash R$. Thus the girth of $\Delta$ being maximal implies the vanishing of $\mathbf{H}_{\operatorname{dim} \Delta}\left(\Delta_{-R}\right)$. Also the vanishing of $\mathbf{H}_{i}(\Delta)$ for $i<\operatorname{dim} \Delta$ except when $i$ is $c-1$ (and $i=-1$ if this homology module is $k$ ), implies the same for $\Delta_{-R}$. Hence $\Delta_{-R}$ is bi-CM of the same dimension and frame dimension as $\Delta$.

Remark 4.5. A slight nuisance in the formulations of Theorems 4.1 and 4.4 is the exception statement. It may be avoided with the following approach. One can sheafify the complexes $L(\Delta)$ to get a complex of coherent sheaves on the projective space $\mathbf{P}^{n}$

$$
\tilde{L}(\Delta): \cdots \rightarrow \mathcal{O}_{\mathbf{P}^{n}}(-p) \otimes_{k}\left(C_{\Delta}\right)_{p} \rightarrow \cdots
$$

and consider the homology sheaves $H_{p+1}(\tilde{L}(\Delta))$ instead of the enriched homology modules $\mathbf{H}_{p}(\Delta)$. Then $H_{0}(\tilde{L}(\Delta))$ is zero when $\mathbf{H}_{-1}(\Delta)$ is $k$ or 0 , and non-zero otherwise, so one avoids the exception in the theorems.

Example 4.6. The boundaries of cyclic polytopes of even dimension $d$ (and containing all vertices of $[n]$ ) are examples of 2-CM designs. Since the boundaries of cyclic polytopes are Gorenstein* they are evidently 2-CM. Furthermore since they are $d / 2$ neighbourly, i.e. $d$ is $2 c, \mathbf{H}_{i}(\Delta)$ vanishes for $i$ in the interval from 0 to $d / 2-2$. By 
Alexander duality $\mathbf{H}_{d-2-i}(\Delta)$ also vanishes when $d-2-i$ is in the interval from $d / 2$ to $d-2$. Hence it is a 2-CM design.

We now turn to examine the rationale behind the term design. Recall that a $t-$ $(n, k, \lambda)$ design is a collection of (distinct) $k$-subsets of $[n]$, called blocks, such that each $t$-subset is contained in exactly $\lambda$ blocks.

Considering the blocks as facets of a simplicial complex $\Delta$ this means that $\Delta$ is pure of dimension $k-1$ and $\mathrm{lk}_{\Delta} T$ has exactly $\lambda$ facets for each $t$-subset $T$. Our $l$ $\mathrm{CM}$ designs will be block designs in that sense. But they have in fact much stronger regularity properties as we show in the following statements.

Lemma 4.7. Let $\Delta$ be a simplicial complex on $[n]$.

a. Suppose that for $x$ in [n] the restrictions $\Delta_{-\{x\}}$ all have the same f-polynomial $f(t)$. Then the f-polynomial $f$ ! of $\Delta$ is given by

$$
f^{!}(t)=\sum_{i=0}^{\operatorname{dim} \Delta+1} \frac{n}{n-i} \cdot f_{i-1} t^{i} .
$$

b. Suppose all links $l k_{\Delta}\{x\}$ have the same f-polynomial $f(t)$. Then the $f$-polynomial $f^{\#}$ of $\Delta$ is given by

$$
f^{\#}(t)=1+n \cdot \sum_{i=0}^{\operatorname{dim} \Delta} \frac{f_{i-1}}{i+1} t^{i+1} .
$$

In particular $f(t)$ is the derivative of $f^{\#}(t)$ divided by the number of vertices.

\section{Proof:}

a. We count the number of pairs $(F, x)$ where $F$ is a face of cardinality $i$ and $x$ is not in $F$. By restricting to each $\Delta_{-\{x\}}$ this is $f_{i-1} \cdot n$. By counting first the $F$ 's in $\Delta$, these pairs can be counted as $f_{i-1}^{!} \cdot(n-i)$.

b. We count the pairs $(F, x)$ where $F$ is a face of cardinality $i+1$ and $x$ is in $F$. Considering each link $\mathrm{lk}_{\Delta}\{x\}$ this is $f_{i-1} \cdot n$. By counting first the $F$ 's, this can be counted as $f_{i}^{\#} \cdot(i+1)$.

Corollary 4.8. Let $\Delta$ be an l-CM design and $Q \subseteq[n]$ with $q \leq l-1$. Then the $f$-vector of $l k_{\Delta} Q$ only depends on $n, d, c, l$, and $q$. In particular, when $Q=\emptyset$, the $f$-vector of $\Delta$ depends only on $n, d, c$, and $l$.

Proof: When $Q=\emptyset$ this follows by repeatedly using Lemma 4.7 a. since all restrictions $\Delta_{-R}$ where $r=l-1$ have the same $f$-vector. If $Q$ is nonempty let $Q=Q^{\prime} \cup\{x\}$. There is a natural exact sequence

$$
0 \rightarrow C_{\mathrm{lk}_{\Delta_{-\{x\}}} Q^{\prime}} \rightarrow C_{\mathrm{lk}_{\Delta} Q^{\prime}} \rightarrow C_{\mathrm{lk}_{\Delta} Q}(-1) \rightarrow 0 .
$$

The statement follows by induction on taking Hilbert series of these modules. 
Corollary 4.9. An l-CM design is an $l-1-(n, d, \lambda)$ block design where $\lambda$ is

$$
\left(\begin{array}{c}
n-d+c+1-l \\
c+1-l
\end{array}\right) \cdot\left(\begin{array}{c}
c \\
l-1
\end{array}\right)^{-1} \cdot\left(\begin{array}{c}
d \\
l-1
\end{array}\right), \quad \text { for } c \geq l-1
$$

and

$$
\left(\begin{array}{c}
n-d \\
l-1-c
\end{array}\right)^{-1} \cdot\left(\begin{array}{c}
l-1 \\
c
\end{array}\right) \cdot\left(\begin{array}{c}
d \\
l-1
\end{array}\right), \text { for } c \leq l-1
$$

\section{Proof:}

a. The restriction $\Delta_{-R}$ when $r=l-1$ is bi-CM with invariants $n+1-l, d$, and $c$ so the number of facets is by (15) given by $\left(\begin{array}{c}n-d+c+1-l \\ c\end{array}\right)$ which we denote by $\lambda^{\prime \prime}$. When $c \geq l-1$ this may be written as

$$
\left(\begin{array}{c}
n-d+c+1-l \\
c+1-l
\end{array}\right) \cdot\left(\begin{array}{c}
c \\
l-1
\end{array}\right)^{-1} \cdot \frac{1}{(l-1) !} \cdot \prod_{i=2}^{l}(n-d+i-l)
$$

and when $c \leq l-1$ this may be written as

$$
\left(\begin{array}{c}
n-d \\
l-1-c
\end{array}\right)^{-1} \cdot\left(\begin{array}{c}
l-1 \\
c
\end{array}\right) \cdot \frac{1}{(l-1) !} \cdot \prod_{i=2}^{l}(n-d+i-l) .
$$

By Lemma 4.7 a. the number of facets of $\Delta_{-R}$ when $r=l-2$ is given by $\lambda^{\prime \prime} \cdot \frac{n+2-l}{n-d+2-l}$ and in general for $r \leq l-1$ by

$$
\lambda^{\prime \prime} \cdot \prod_{i=2}^{l-r} \frac{n+i-l}{n-d+i-l}
$$

The expression for this when $r=0$ will be the number of facets of $\Delta$ and we denote it by $\lambda^{\prime}$. Now by Lemma $4.7 \mathrm{~b}$. the number of facets of $\operatorname{lk}_{\Delta} Q$ when $q=1$ is $\lambda^{\prime} \cdot \frac{d}{n}$ and in general for $q \leq l-1$ it will be

$$
\lambda^{\prime} \cdot \prod_{i=0}^{q-1} \frac{d-i}{n-i}
$$

When $q=l-1$ this is

$$
\lambda=\lambda^{\prime} \cdot \prod_{i=2}^{l} \frac{d+i-l}{n+i-l} .
$$

By working out the result of these steps starting from (18) and (19) we obtain the statement. 
Example 4.10. According to Conjecture 2.8 the minimal interesting value of $c$ for $l$-CM designs is $l-1$. And then the minimal interesting value for $d$ is $l$. By the above we see that $\lambda=l$ so we get $l-1-(n, l, l)$ designs. Such designs have been constructed when $l=3$ [18], and $l=4$ [15], in many cases. It is not automatic that they are $l$-CM designs but we suspect that many of the examples constructed are.

A consequence of the Corollary 4.9 is that it supports Conjecture 2.8.

Corollary 4.11. There is a function $\sigma(l, d)$ such that if $\Delta$ is an l-CM design with $n \geq \sigma(l, d)$ then $c \geq l-1$.

Proof: The expression of (17) is for $c \leq l-2$ the expression of (17) is equal to

$$
\frac{d(d-1) \cdots(d+2-1)}{c !(n-d) \cdots(n-d+2+c-l)} .
$$

If this is an integer then

$$
\frac{d(d-1) \cdots(d+2-l)}{n-d}
$$

is an integer and so the statement follows.

\section{3 (a, b)-designs}

Now we introduce a class of designs which extends the class of $l$-CM designs. Call a simplicial complex an $(a, b)$-design if

$$
\left(\mathrm{lk}_{\Delta} B\right)_{-A}
$$

is bi-CM of dimension and frame dimension equal to those of $\Delta$ reduced by $b$, whenever $A$ and $B$ are disjoint subsets of $[n]$ of cardinalities $a$ and $b$. Observe that the Alexander dual of an $(a, b)$-design is a $(b, a)$-design, since the Alexander dual of (20) is $\left(\mathrm{lk}_{\Delta^{*}} A\right)_{-B}$. Note also that $l$-CM designs are $(l-1,0)$-designs and so $(0, l-1)$ designs are Alexander duals of $l$-CM designs.

Example 4.12. The standard triangulation of the real projective plane with six vertices, and the triangulation of the two-dimensional torus with seven vertices are examples of (1,1)-designs $\Delta$ with $d=3$ and $c=2$. This is because each link $\operatorname{lk}_{\Delta}\{x\}$ is a polygon with $n-1$ vertices and invariants $n-1, d-1$, and $c-1$. Thus it is a (1,0)-design (or 2-CM design) with the right invariants. Note that both reduced homology groups vanish for the real projective plane (char $k \neq 2$ ) while the torus has one-dimensional $\tilde{H}_{2}(\Delta)$ and two-dimensional $\tilde{H}_{2}(\Delta)$.

It is known for which genera of orientable and non-orientable surfaces there exist 2-neighbourly triangulations, see [14, 21]. These will be examples of $(1,1)$-designs.

In general $(1,1)$-designs with $d=2 c-1$ will be homology manifolds since each $\mathrm{lk}_{\Delta}\{x\}$ gives a $(1,0)$-design (or 2-CM design) with invariants $d-1$ and $c-1$ where 祭 Springer 
$d-1=2(c-1)$. Conferring Example 4.6 one may work out, via Euler characteristics, that the rank of the top cohomology module of each single-point link is one, and hence each of these links are Gorenstein* by Theorems 2.1 and 3.1. Such homology manifolds are related to certain extremal behaviour of the Euler characteristic, see [16, 17-20] for a survey.

We now show that $(a, b)$-designs have very strong regularity properties. Not only is the number of facets of links determined, but the complete $f$-vector of all combinations of links and restrictions up to a certain level is determined.

Lemma 4.13. Let $\Delta$ be an $(a, b)$-design and $R$ and $Q$ be disjoint subsets of $[n]$ where $r+q \leq a+b$. Then the $f$-vector of

$$
\left(l k_{\Delta} Q\right)_{-R}
$$

depends only on the numerical invariants $n, d, c, a, b, r$, and $q$.

Proof: If $Q$ and $R$ are empty this follows by repeated use of Lemma 4.7. Suppose then that $r \leq a$ and $q \leq b$. Then (21) is an $(a-r, b-q)$-design and so this follows by the case just treated. If $q>b$ then let $B \subseteq Q$ be $a$ subset of cardinality $b$. Then $\left(\mathrm{lk}_{\Delta} B\right)_{-R}$ is a $(a-r, 0)$-design. Since (21) is a further link of this we get the statement from Corollary 4.8. If $r>a$ we may reduce to the case just considered by taking the Alexander dual.

Remark 4.14. Considering the case when $Q$ has cardinality $a+b$ we see that $(a, b)$ designs are $a+b-(n, d, \lambda)$ block designs for some $\lambda$. By taking the link of a set of cardinality $b$ we get an $(a, 0)$-design which is an $a-(n-b, d-b, \lambda)$ design with invariants $n-b, d-b$ and $c-b$. Hence $\lambda$ may be determined by Corollary 4.9.

Example 4.15. A consequence of the above remark is that $(0, l-1)$-designs have $\lambda$ equal to $\left(\begin{array}{c}n+1+c-d-l \\ c+1-l\end{array}\right)$. Hence when $c=l-1$ we get $c-(n, d, 1)$ designs and these are exactly the Steiner systems $S(c, d, n)$. They are Alexander duals of the $l$-CM designs where $d$ is submaximal equal to $n-l$.

\section{Problems and conjectures}

We pose the following problems.

Problem 1. What are the possible $f$-vectors (or $h$-vectors) of $l$-Cohen-Macaulay simplicial complexes?

The case when $l$ is equal to 1 is classical, see [24, II.3.3]. When $l \geq 2$ this is likely to be a difficult problem since any answer also would include a question of what the $h$-vectors of Gorenstein* simplicial complexes are. However, any conjecture about this would be highly interesting since it would contain as a subconjecture what the $h$-vectors of Gorenstein* simplicial complexes are. Some investigations into this problem are contained in [25]. 
This problem might be more tractable if $d$ is an extremal value.

Subproblem 2. What are the possible $f$-vectors (or $h$-vectors) of $l$-CM simplicial complexes with $d$ equal to $n-l$ ?

Problem 3. Construct ( $a, b)$-designs for various parameters of $n, d, c, a$, and $b$.

As has been pointed out this has been done in a number of particular cases. When $a$ and $b$ are zero we have the bi-Cohen-Macaulay simplicial complexes constructed in [10]. When $a$ is $1, b$ is 0 and $d$ is $2 c$ we have the cyclic polytopes. When $a$ and $b$ are 1, triangulations of surfaces has been constructed. For $a=0$, low values of $b$ and $c=b$ many Steiner systems $S(c, d, n)$ have been constructed. An examination of the literature on designs will most likely reveal numerous other cases.

Theorem 4.4 and Example 4.12 also suggest the following.

Problem 4. Determine the homological behaviour of $(a, b)$-designs $\Delta$. For instance do the dimensions of $\tilde{H}_{i}(\Delta)$ depend only on $n, d, c, a$, and $b$, and if so, what are they?

We also recall the following from Section 2.

Conjecture 2.8. Let $\Delta$ be an $l$-CM simplicial complex of maximal girth. Assume it is not the $r$-skeleton of the $l+r-1$-simplex for some $r$. Then $c \geq l-1$.

A weaker form of this conjecture is

Conjecture 5.1. Show that there is an integer $\sigma(l, d)$ such that if $\Delta$ is an $l$-CM simplicial complex of maximal girth where the number of vertices is larger than this integer, then $c \geq l-1$.

Acknowledgments I thank an anonymous referee for suggestions simplifying the proofs of Theorems 3.1 and 3.4, and in general the referees for improving the exposition of the paper.

\section{References}

1. K. Baclawski, "Cohen-Macaulay connectivitiy and geometric lattices," Europ. J. Combinatorics 3 (1982), 293-305.

2. D. Bayer, I. Peeva, and B. Sturmfels, "Monomial resolutions," Math. Res. Lett. 5(1-2) (1998), 31-46.

3. D. Bayer and B. Sturmfels, "Cellular resolutions of monomial ideals," J. Reine Angew. Math. 102 (1998), 123-140.

4. W. Bruns and J. Herzog, "Cohen-Macaulay rings," Cambridge Studies in Advanced Math., Cambridge University Press (1993) vol. 39.

5. D. Eisenbud, G. Fløystad, and F.-O. Schreyer, "Sheaf cohomology and free resolutions over exterior algebras," Trans. Am. Math. Soc. 355(11) (2003), 4397-4426.

6. G. Fløystad and J.E. Vatne, "(Bi)-Cohen-Macaulay simplicial complexes and their associated coherent sheaves," Commun. Alg. 33(9) (2005), 3121-3136.

7. G. Fløystad, "Cohen-Macaulay cell complexes," preprint, math. CO/0502541, to appear in Proceedings, Algebraic and Topological Combinatorics, Anogia, 2005.

글 Springer 
8. M. Hochster, "Cohen-Macaulay rings, combinatorics, and simplicial complexes," in B.R. McDonald and R. Morris (Ed.), Ring Theory II, Proc. Second Oklahoma Conference, Dekker, New York, 1977, pp. 171-223.

9. M. Jungerman and G. Ringel, "Minimal triangulations on orientable surfaces," Acta Math. 145 (1980), $121-154$.

10. D.L. Kreher, "t-Designs, $t \geq 3$, the CRC handbook of combinatorial designs," in Charles Colbourn and Jeffrey Dinitz (Eds.), the CRC Handbook of Combinatorial Designs (1996), pp. 47-65.

11. W. Kühnel, "Triangulations of manifolds with few vertices," in F. Tricerri (Ed.), Advances in Differential Geometry and Topology, World Scientific, Singapore, 1990, pp. 59-114.

12. F.H. Lutz, "Triangulated manifolds with few vertices: Combinatorial manifolds," preprint, http://arxiv.org/math.CO/0506316.

13. R. Mathon and A. Rosa, "2 - $(v, k, \lambda)$ Designs of small order," in Charles Colbourn and Jeffrey Dinitz (Eds.), The CRC Handbook of Combinatorial Designs, CRC Press (1996), pp. 3-41.

14. E. Miller, "Alexander duality functors and local duality with monomial support," J. Alg. 231 (2000), 180-234.

15. I. Novik, "Upper bound theorems for homology manifolds," Isr. J. Math. 108 (1998), 45-82.

16. G. Ringel, "Wie man die geschlossene nichtorientierbaren Flächen in möglichst wenig Dreiecke zerlegen kann," Maht. Ann. 130 (1955), 317-326.

17. T. Römer, "Generalized Alexander duality and applications," Osaka J. Math. 38(2) (2001), 469-485.

18. P. Schenzel, "On the number of faces of simplicial complexes and the purity of Frobenius," Math. Zeit. 178 (1981), 125-142.

19. R. Stanley, Combinatorics and Commutative Algebra, 2nd edition, Birkhäuser, 1996.

20. E. Swartz, "Lower bounds for h-vectors of k-CM, independence and broken circuit complexes," SIAM J. Discrete Math. 18 (2004), 647-661.

21. J. Stückrad and W. Vogel, Buchsbaum Rings and Applications, Springer Verlag, 1986.

22. K. Yanagawa, "Alexander duality for Stanley-Reisner rings and squarefree $\mathbf{N}^{n}$-graded modules," $J$. Algebra 225 (2000), 630-645.

23. G. Ziegler, Lectures on Polytopes, GTM 152, Springer-Verlag, 1995. 